\title{
Estrategias de comprensión lectora y producción textos argumentativos en estudiantes estudios generales de una universidad privada - Lima
}

Jorge Roncal Vargas ${ }^{1}$

jroncal6@gmail.com

\author{
Abelardo Rodolfo Campana Concha ${ }^{2}$ \\ abel5454@hotmail.com \\ Orcid: $\underline{0000-0002-1098-9508}$
}

\section{RESUMEN}

Objetivo: Analizar la influencia de la aplicación de estrategias de comprensión lectora en la producción de textos argumentativos en estudiantes universitarios. Materiales $\mathbf{y}$ Método: Es un estudio cuasiexperimental que contó con un grupo control y experimental a una muestra de 99 estudiantes, que será procesada con el programa SPSS. Resultados: Se comprobó la estrategia aplicada mejoró la dependiente, produciéndose diferencia de 4.03 puntos entre GC y GE. hipótesis específicas de igual modo como: manejo de fuentes, construcción de párrafos jerárquicamente, estrategias discursivas en la organización de las ideas, revisión y corrección de la redacción, las medias entre están entre: 2.36, 2.78, 2.22 y 3.13 puntos, respectivamente. Conclusión: se incrementó 4.03 en el promedio entre GC y GE, encontrándose están en "proceso" y logrado, demuestran que a los estudiantes les resulta favorable la aplicación de la estrategia empleada para producir textos argumentativos.

Palabras clave: estrategia; comprensión lectora; producción; texto argumentativo.

\footnotetext{
${ }^{1}$ Estudiante de Maestría en Educación de la Universidad Nacional Mayor de San Marcos

${ }^{2}$ Docente de Educación de la Universidad Nacional Mayor de San Marcos
} 


\title{
Strategies for reading comprehension and production of argumentative texts in general studies students from a private university - Lima
}

\begin{abstract}
Objective: To analyze the influence of the application of reading comprehension strategies in the production of argumentative texts in university students. Materials and Method: It is a quasi-experimental study that included a control and experimental group with a sample of 99 students, which will be processed with the SPSS program. Results: The applied strategy was found to improve the dependent one, producing a difference of 4.03 points between CG and EG. Specific hypotheses in the same way as: handling of sources, construction of paragraphs hierarchically, discursive strategies in the organization of ideas, revision and correction of the writing, the means between are between: $2.36,2.78,2.22$ and 3.13 points, respectively. Conclusion: there was an increase of 4.03 in the average between CG and GE, being found to be in "process" and achieved, they show that the students are favourable to the application of the strategy used to produce argumentative texts.
\end{abstract}

Keywords: strategy; reading comprehension; production; argumentative text

Artículo recibido: 02 noviembre. 2021 Aceptado para publicación: 28 noviembre 2021

Correspondencia: jroncal6@gmail.com Conflictos de Interés: Ninguna que declarar 


\section{INTRODUCCIÓN}

Las evaluaciones internaciones como el PISA, nos dan una noción de la realidad lectora en el país, según el Ministerio de Educación (MINEDU, 2018) en estas evaluaciones, los estudiantes peruanos alcanzaron 401 puntos en la comprensión lectora, incrementándose solo 3 con respecto a la realizada en el 2015, incluso estamos en ubicados en las últimas ubicaciones a nivel Sudamérica. Es decir, el estudiante termina su etapa escolar con un nivel lector bajo, lo cual creará dificultades a futuro cuando empiece su vida universitaria. Lo anterior señalado es reforzado por las opiniones de Ramos (2011) quien señala que los estudiantes universitarios empiezan esta vida académica con los hábitos escolares reflejados en la copia de textos, sin evidenciar un vínculo entre la lectura y la escritura, además agrega que:

[...] la universidad peruana se ha visto obligada a reconocer el problema y, por ello, en las últimas décadas, ha incorporado cursos de redacción o argumentación en sus primeros ciclos; es decir, al confirmar que los alumnos no pueden formalizar sus pensamientos en un texto, se ha visto obligada a establecer asignaturas escolares en su currículo, que ocupan espacios en los que deberían ofrecerse otros cursos de la propia carrera (p.9).

A pesar que el estudiante universitario no haya recibido el adecuado desarrollo de su habilidad lectora, es importante entender el compromiso universidad como se señala en la Ley N³0220 la cual es la de "formar profesionales de alta calidad de manera integral y con pleno sentido de responsabilidad social de acuerdo a las necesidades del país".

Por ello, como docentes es compromiso nuestro ayudar a los estudiantes en este proceso lector mediante las estrategias que ayuden de forma efectiva para analizar lo que lee en beneficio propio para crear sus propios textos, ya que en la etapa universitaria los estudiantes tendrán que realizar trabajos universitarios entre ensayos, monografías o exámenes, tendrán que aprender a sustentar sus argumentos.

Por su parte, Ferrucci y Pastor (2013) agregan que "los fundamentos básicos de la redacción de textos académicos deberían estar aprendidos" (p.21) con la finalidad de que puedan incluir en sus redacciones coherencia solidez y organización. Curiosamente, estos puntos son propios y necesarios para redacción argumentativa, sin embargo, la ausencia de herramientas cognitivas e interactivas para leer y escribir hacen notoria la problemática 
de los estudiantes en los distintos niveles académicos (Araya y Roig, 2014).

Existen antecedentes que también abordaron esta problemática, como los de Díaz (2020) quien confirma la influencia positiva de las estrategias de lectura, ya que el promedio de su muestra aumentó significativamente de 14,69 (prueba pre test) a 17,31 (prueba postest), repitiéndose resultados similares en el literal, inferencial y criterial. Por ello, considera necesario "capacitar a los estudiantes en el conocimiento de los diversos tipos de estrategias de aprendizaje para desarrollar las habilidades en los estudiantes" (p.76). Por otro lado, tenemos a Cortez (2021) quien concluye que el nivel académico para la producción de textos de este tipo no se desarrolla adecuadamente debido a la metodología convencional, generando desinterés por parte del alumnado ya que no puedan exponer sus ideas y sustentarlas, evitando así autonomía en su aprendizaje y que sean meros receptores y no personas críticas de la realidad que los rodea.

Por tanto, el objetivo de este estudio es analizar la influencia la aplicación de estrategias de comprensión lectora en la producción de textos argumentativos en estudiantes área humanidades de estudios generales - UTP, 2020.

\section{MÉTODO}

Corresponde al experimental, diseño "cuasi experimental”, compara GC y GE, la muestra es representada por 99 de estudiantes. Se les aplicó el pre y pos test procesados estadísticamente con el T Student.

\section{RESULTADOS}

Tabla 1. Frecuencias pretest variable dependiente

\begin{tabular}{llcc}
\hline & & Frecuencia & Porcentaje \\
\hline \multirow{3}{*}{ Válidos } & Básico & 37 & 37,4 \\
& Proceso & 62 & 62,6 \\
\cline { 2 - 4 } & Total & $\mathbf{9 9}$ & $\mathbf{1 0 0 , 0}$ \\
\hline
\end{tabular}

Figura 1. Porcentajes pretest variable dependiente (VD)

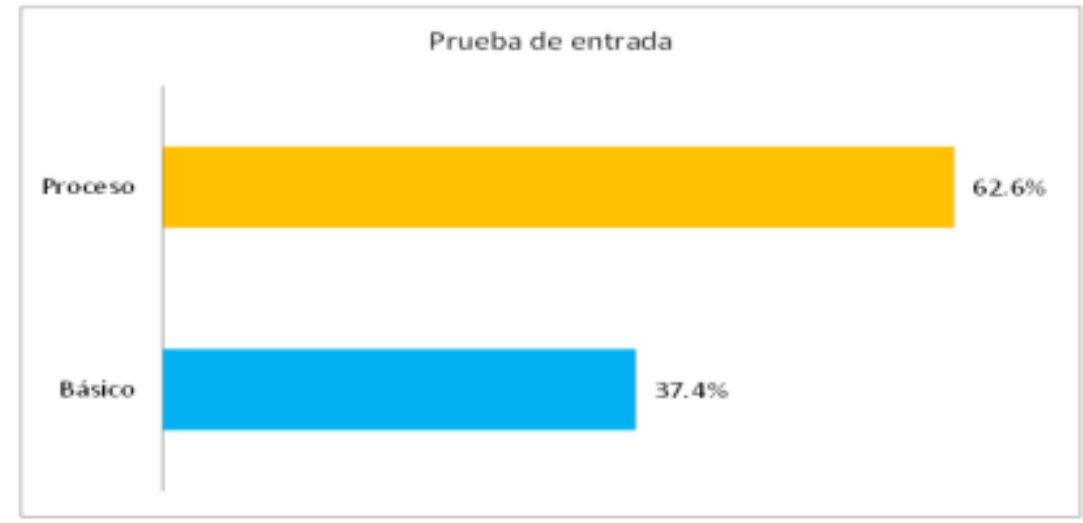


Antes de aplicar estrategias de comprensión de lectura, el 62,6\% alcanzó el nivel en proceso en la producción de textos argumentativos y el 37,4\% un nivel básico.

Tabla 2, Frecuencias postest variable dependiente (VD)

\begin{tabular}{llcc}
\hline & & Frecuencia & Porcentaje \\
\hline \multirow{3}{*}{ Válidos } & Proceso & 25 & 25,3 \\
& Logrado & 74 & 74,7 \\
\cline { 2 - 4 } & Total & $\mathbf{9 9}$ & $\mathbf{1 0 0 , 0}$ \\
\hline
\end{tabular}

Figura 2. Porcentajes postest variable dependiente

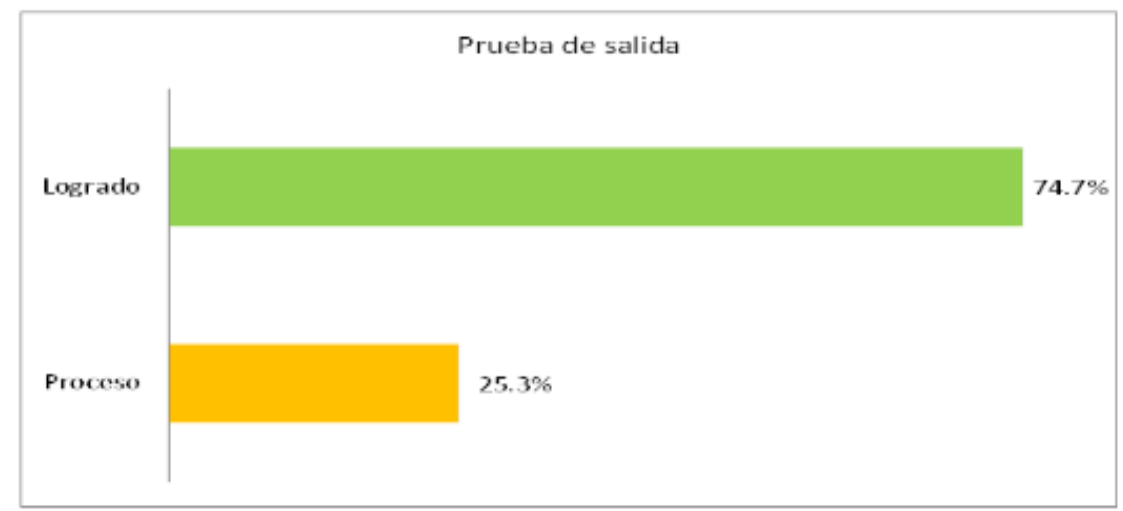

Luego de aplicar las estrategias de comprensión lectora, el 74,7\% alcanzan un nivel logrado mientras que el 25,3\% el nivel proceso, por lo cual en el postest se evidencia un avance por parte de los estudiantes.

\section{Prueba hipótesis general}

HG. La aplicación de estrategias de comprensión lectora mejora en la producción de textos argumentativos en estudiantes área humanidades de estudios generales - UTP, 2020.

Tabla 3. Media VD

\begin{tabular}{|c|c|c|c|c|}
\hline \multicolumn{5}{|c|}{ Muestras relacionadas } \\
\hline $\begin{array}{l}\text { Grupo de } \\
\text { estudio }\end{array}$ & Media & $\mathrm{N}$ & Desviación típ. & Error típ. de la media \\
\hline Control & 10,79 & 99 & 1,372 & , 138 \\
\hline Experimental & 14,82 & 99 & 1,722 &, 173 \\
\hline
\end{tabular}


En las medias del grupo control (GC) se evidencia un promedio de 10,79, mientras que posterior a la aplicación de la estrategia de comprensión lectora, el grupo experimental (GE) obtuvo un promedio de 14,82, es decir se incrementó en 4.03, lo cual comprueba que esta estrategia facilita la producción de textos argumentativos.

\section{Prueba hipótesis específica 1}

H1. La aplicación de estrategias mejora el Manejo de fuentes.

Tabla 4. Medias HE1

\begin{tabular}{|c|c|c|c|c|c|}
\hline \multirow{3}{*}{ 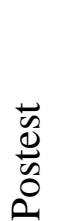 } & Grupo & $\mathbf{N}$ & Media & Desviación & Error típ. \\
\hline & Control & 36 & 13,08 & 1,204 & ,201 \\
\hline & Experimental & 63 & 15,44 & 1,511 & , 190 \\
\hline
\end{tabular}

En grupo control se observó una media de 13,08, por su lado la media experimental con puntaje de 15,44, es decir los 2,36 de diferencia evidencia que luego de aplicarse la estrategia de lectura el estudiante incrementa sus conocimientos en el manejo de fuentes.

\section{Prueba hipótesis específica 2}

H2. "La aplicación de estrategias de comprensión lectora mejoró la construcción de párrafos jerárquicamente".

Tabla 3, Diferencia de medias HE2

\begin{tabular}{|c|c|c|c|c|c|c|}
\hline \multirow{3}{*}{ 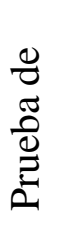 } & & Grupo & $\mathbf{N}$ & Media & Desviación & Error típ. \\
\hline & \multirow{2}{*}{ 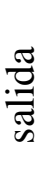 } & Control & 49 & 13,00 & ,935 & ,134 \\
\hline & & Experimental & 50 & 15,78 & 1,620 & ,229 \\
\hline
\end{tabular}

Los resultados de la media en el grupo control $(13,00)$ y el grupo experimental $(15,78)$ confirman que las aplicaciones de estrategias de comprensión lectora incrementan sus conocimientos para la construcción de párrafos jerárquicamente, debido al incremento de 2,78 .

\section{Prueba hipótesis específica 3}

H3. La aplicación de estrategias de comprensión lectora mejora las estrategias discursivas en la organización de las ideas. 
Tabla 4. Diferencia media $H 3$

\begin{tabular}{|c|c|c|c|c|c|}
\hline \multirow{3}{*}{ 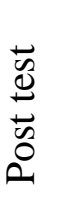 } & Grupo & $\mathbf{N}$ & Media & Desviación & Error típ. \\
\hline & Control & 49 & 13,16 & 1,313 & , 188 \\
\hline & Experimental & 50 & 15,38 & 1,563 & ,221 \\
\hline
\end{tabular}

La media del grupo control evidencia un promedio de 13,16, sin embargo, luego de aplicar la estrategia de comprensión lectora, el grupo experimental obtuvo un promedio de 15,38, es decir se incrementó en 2,22, lo cual comprueba que esta estrategia ayuda a los estudiantes a establecer estrategias discursivas en la organización de las ideas.

\section{Prueba hipótesis específica 4}

H4. "La aplicación de estrategias de comprensión lectora mejora la revisión y corrección de la redacción".

Tabla 5. Diferencia media HE4

\begin{tabular}{|c|c|c|c|c|c|}
\hline \multirow{3}{*}{ 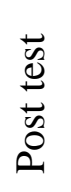 } & Grupo & $\mathbf{N}$ & Media & Desviación & Error típ. \\
\hline & Control & 49 & 12,73 & ,884 & , 126 \\
\hline & Experimental & 50 & 15,86 & 1,340 & , 190 \\
\hline
\end{tabular}

En el grupo control se observó una media de 12,73, por su lado la media experimental con puntaje de 15,86, es decir los 3,13 de diferencia evidencia que luego de aplicarse la estrategia de lectura el estudiante incrementa sus conocimientos para la revisión y corrección de la redacción.

\section{DISCUSIÓN DE LOS RESULTADOS}

En la hipótesis general se obtuvo en el grupo control un promedio de 10,79, cuya mayoría alcanzó calificaciones en "proceso"; en tanto que, en el grupo experimental, una media de 14,82, con promedios mayoritarios en "logrado", en otros términos, con la aplicación de estrategias de comprensión lectora los estudiantes consiguieron un incremento en sus promedios en las actividades relacionadas a la producción textos argumentativos.

En cuanto al planteamiento de la hipótesis específica $\mathrm{n}^{\circ} 1$, las estadísticas encontraron una media de 13,08 GC, y GE 15,44, los promedios mayoritarios alcanzaron de básico al nivel logrado, respectivamente. Evidenciando que la muestra experimentó una influencia 
positiva con la aplicación de las estrategias de comprensión de lectura para el manejo de fuentes.

En la hipótesis específica $\mathrm{n}^{\circ} 2$, las estadísticas establecieron una media de 13,00 GC y 15,78 GE, la muestra logró influenció positivamente en la construcción de párrafos jerárquicamente, con la aplicación de las estrategias de comprensión lectora, debido a que los promedios de la muestra mostraron un crecimiento al pasar de calificaciones mayoritarias en "proceso" a "logrado".

En las estadísticas que responden al planteamiento de la hipótesis 3, se encontró una media de 13,16 GC, mientras GE 15,38, donde los estudiantes destacaron por sus promedios mayoritarios de nivel básico y nivel logrado, respectivamente. Entendiendo que la muestra experimentó una influencia favorable con la aplicación de las estrategias de comprensión de lectura para el Uso de estrategias discursivas en la organización de las ideas.

En la hipótesis específica ${ }^{\circ} 4$, los resultados estadísticos muestran media de 12,73 GC y 15,86 GE, es decir los estudiantes lograron establecer un aceptable progreso en la revisión y corrección de la redacción, con la aplicación de las estrategias de comprensión lectora, a su vez manifestado en los promedios de la muestra quienes evidenciaron un crecimiento al pasar de calificaciones mayoritarias en "básico" a "logrado".

Realizando el contraste de nuestros resultados con estudios anteriores, encontramos los de Paucara (2018) quien considera que la comprensión lectora debe ser entendida desde una perspectiva diversa por la existencia de factores históricos, culturales, económicos, etc. por ello mientras más se lee mayor será comprensión, sin embargo considera que no debe desarrollarse mecánicamente, sin embargo esta mala costumbre se puede ir disminuyendo con la práctica pero con una adecuada, demostrado en nuestros resultados cuyas estrategias en la comprensión lectora, no solo permitió el mejoramiento en la lectura, sino en la producción de textos argumentativos, pasando de un nivel básico a un nivel logrado.

Asimismo, tenemos las investigaciones de Camacho (2017) quien considera que las estrategias para redactar ayudan a los estudiantes a producir textos argumentativos, cuyo grupo control alcanzó el nivel en inicio y el grupo experimental como nivel logrado. Sin embargo, hay que tener cuenta que no solo se necesita que el estudiante conozca técnicas para redactar, sino estrategias lectoras, porque la lectura es la que lo ayudará ampliar sus 
conocimientos para sustentar sus ideas al momento de redactar, lo cual lo hemos demostrado en nuestros resultados, ya que nuestra muestra alcanzó el nivel logrado, aplicando estrategias de comprensión lectora, por ello es importante entender que el leer y escribir trabajan en conjunto.

\section{CONCLUSIONES}

1. El incremento de 4.03 en el promedio entre el grupo control y experimental, respectivamente, demuestran que a los estudiantes les resulta favorable la aplicación de estrategias de comprensión lectora para la producción de textos argumentativos.

2. Los estudiantes del grupo control alcanzaron un nivel básico, sin embargo, luego de aplicar las estrategias de compresión lectora, el grupo experimental alcanzó el nivel logrado, evidenciado además en el incremento de 2,36 entre ambos grupos, que confirma la eficacia de esta estrategia en el manejo de fuentes.

3. La mayoría de los estudiantes del grupo control están en un nivel básico en la construcción de párrafos jerárquicamente, sin embargo, luego de aplicar la estrategia de comprensión lectora, el grupo experimental obtuvo el nivel logrado, con un incremento de 2,78 en su promedio, confirmando la eficacia para que el estudiante pueda estructurar la introducción de texto, conectarlo párrafo a párrafo, establecer la correcta puntuación y ortografía.

4. Las estrategias de comprensión lectora resultan efectivas para los estudiantes de educación superior para el uso de las estrategias discursivas en la organización de las ideas, debido a que la mayoría del grupo control alcanzó el nivel básico, en tanto que los estudiantes del grupo experimental alcanzaron un nivel logrado, aumentando su promedio en 2,22 puntos, demostrando así que la muestra mejoró en la solidez de su contenido textual, el uso de conectores discursivos, explicación causal y la ejemplificación.

5. Resulta favorable aplicar las estrategias de comprensión lectora en estudiantes universitarios, debido a que consiguieron incrementar su promedio en 3,13 puntos en lo referente a la revisión y corrección de la redacción, considerando que la mayoría de los estudiantes alcanzó un nivel básico (grupo control) y nivel logrado (grupo experimental), evidenciando un mejoramiento para la elaboración de cada parte del texto, jerarquizar ideas, uso de conectores y referentes. 


\section{AGRADECIMIENTOS}

Dedicado al apoyo incondicional de mis padres: Jorge y Carolina

\section{REFERENCIAS BIBLIOGRAFICAS}

Camacho, M. (2017). Estrategias para redactar en la producción de textos argumentativos en estudiantes de quinto grado de secundaria [Tesis Magisterial, Universidad Cesar Vallejo].

Cortez, J. (2021). Nivel de dominio de la producción de textos argumentativos en el segundo grado de educación secundaria en una institución educativa de Chiclayo [Tesis de Licenciatura, Universidad Católica Santo Toribio de Mogrovejo].

De Lera, P. (2017). Estudio de la instrucción en comprensión lectora: ámbito educativo y científico [Tesis Doctoral, Universidad de León].https://buleria.unileon.es/handle/10612/6941

Domínguez, M. (2009). Análisis de la coherencia en los textos producidos en clases de Física en el nivel polimodal. Revista Iberoamericana de Educación, 48 (3), 1-11. https://rieoei.org/historico/deloslectores/2522Dominguez.pdf

Ferrucci, G. y Pastor, C. (2013). Desarrollo alcanzado en la redacción académica por los alumnos ingresantes a u curso de habilidades lingüísticas básicas de una universidad privada de Lima [Tesis de Maestría, PUCP].

Gernsbacher, M. (1990). Language comprehension as structure building. Lawrence Erlbaum Associates, Inc.

González, A. (2004). Manual de redacción. Editorial Universitaria de la Universidad Ricardo Palma.

Ley 1060 de 2014. Ley universitaria. 9 de julio de 2014.

López, M. (2005). Estrategias de comprensión. Ediciones de la UCLM.

López, M. (2005). Estrategias de comprensión. Ediciones de la Universidad de CastillaLa Mancha.

Ministerio de Educación (2018). Evaluación PISA 2018. https://es.calameo.com/read/006286625977c1ced4d6c?view=slide\&page=1

Molina, J. (2019). Producción de textos argumentativos a través de la multimedia en los estudiantes del grado $9^{\circ} \mathrm{D}$ de la I.E. La Salle de Campoamor [Tesis de Licenciatura, Universidad Cooperativa de Colombia]. 
https://repository.ucc.edu.co/bitstream/20.500.12494/10963/2/2019_produccion_ textos_argumentativos.pdf

Obando, L. (2013). Marco epistemológico para el desarrollo de la competencia argumentativa escrita. Revista de la Educación Colombiana, 16 (16), 343-368.

Paucara, M. (2018). La práctica lectora y su incidencia en la comprensión de la lectura caso: Jóvenes y adultos del 5to. y 6to. de secundaría "A" del Centro de Educación Alternativa Politécnico Corea [Tesis de Licenciatura, Universidad Mayor de San Andrés].

Parodi, G. (2000). La evaluación de la producción de textos escritos argumentativos: una alternativa cognitiva/discursiva. Revista Signos, 33(47), 151-166. https://www.scielo.cl/scielo.php?script=sci_arttext\&pid=S071809342000000100012

Parodi, G., Peronard, M. y Ibáñez, R. (2010). Saber leer. Santillana.

Ramos, M. (2011). El problema de comprensión y producción de textos en el Perú. Docencia Universitaria, 5 https://revistas.upc.edu.pe/index.php/docencia/article/view/5/153

Serrano, S. (2008). Composición de textos argumentativos. Una aproximación didáctica. Revista de Ciencias $\quad$ Sociales, $14 \quad$ (1), p.149161.https://www.redalyc.org/pdf/280/28011673013.pdf

Solé, I. (2001). Estrategias de lectura. Barcelona: Graó

Van Dijk, T. y Kintsch, W. (1983). Strategies of discourse comprehension. Academic Press 